\title{
A BOUNDED ANALYTIC FUNCTION IN THE UNIT DISK WITH A LEVEL SET COMPONENT OF INFINITE LENGTH
}

\author{
K. F. BARTH AND J. G. CLUNIE ${ }^{1}$ \\ Dedicated to the memory of David L. Williams
}

\begin{abstract}
The authors construct a bounded analytic function in the unit disk with a level set component of infinite length. The example is of the form $\exp i B(z)$ where $B(z)$ is a Blaschke Product.
\end{abstract}

1. Introduction. In [6] Piranian and Weitsman showed that bounded analytic functions in the unit disk can have level sets of infinite length. Subsequently a number of authors have obtained results of this kind [1, 5, 3]. In their paper Piranian and Weitsman raise the question as to whether or not a function analytic and univalent in the unit disk can have a level set of infinite length. Recently Hayman and $\mathrm{Wu}$ [3] have shown that this is not possible. Another proof of this result has been given by Garnett, Gehring and Jones [2].

The examples in the above papers of bounded analytic functions with level sets of infinite length have disconnected level sets whose components are all of finite length apart, perhaps, from that of Peter Jones [4]. Whether or not the construction of Peter Jones leads to an example with a level set component of infinite length is not known and his proof gives no indication one way or the other.

In this paper we shall give an explicit construction of a bounded analytic function having a level set component of infinite length. That the component is of infinite length is due to its being highly branched and the case of an unbranched component of infinite length is still unsettled. There are many examples of unbounded holomorphic functions with an unbranched level set component of infinite length. One such function is the following: Let $S_{1}$ and $S_{2}$ be two disjoint spirals each of which is contained in $D \equiv\{|z|<1\}$ and tends to all of $C \equiv\{|z|=1\}$. Then, using standard approximation techniques, one can construct a function $f$, holomorphic in $D$ with nonzero derivative, that approaches infinity on $S_{1}$ and zero on $S_{2}$ as $|z| \rightarrow 1$. It follows easily that every level set $L(\lambda, f) \equiv\{z:|f(z)|=\lambda\}, 0<\lambda<\infty$, has an unbranched component of infinite length.

Received by the editors October 5, 1981 and, in revised form, January 4, 1982. Presented to the Summer Meeting of the American Mathematical Society, Pittsburgh, Pennsylvania, August 19, 1981.

1980 Mathematics Subject Classification. Primary 30D50; Secondary 30D40.

Key words and phrases. Bounded functions, level set, level curve.

'The authors gratefully acknowledge support from the National Science Foundation. 
2. A bounded analytic function with a level set component of infinite length.

THEOREM 1. There exists a function $f$, analytic and bounded in the unit disk, such that $L(1, f)$ has a component of infinite length.

The example is of the form $f(z)=\exp (i B(z))$ where $B(z)$ is a Blaschke product of the form $\prod_{v=1}^{\infty}\left[\left(\rho_{v}-z\right) /\left(1-\rho_{v} z\right)\right]^{n_{v}}$ and $0<\rho_{v}<1$. Since $|f(z)|=1$ exactly when $B(z)$ is real, it suffices to estimate the length of the set in the unit disk on which $B(z)$ is real. This is accomplished by a sequence of lemmas.

LEMMA 1. Let $\rho$ and $\theta$ be real numbers satisfying $\frac{1}{4} \leqslant \rho<1,0<\theta \leqslant \pi / 2$, and $\sin \theta / 2 \geqslant 1-\rho$. Then

$$
d(\theta) \equiv \frac{\left(1-\rho^{2}\right) \sin \theta}{\left|1-\rho e^{i \theta}\right|^{2}}
$$

satisfies

$$
\frac{1-\rho}{4 \theta} \leqslant d(\theta) \leqslant \frac{4 \pi(1-\rho)}{\theta}
$$

Proof. First we note that

$$
d(\theta)=\frac{2\left(1-\rho^{2}\right) \sin \frac{\theta}{2} \cos \frac{\theta}{2}}{\left(1-\rho^{2}\right)+4 \rho \sin ^{2} \frac{\theta}{2}}
$$

and then use the inequalities $0 \leqslant \rho<1$ and $\cos \theta / 2 \geqslant 1 / \sqrt{2}$ to obtain

$$
d(\theta) \geqslant \frac{(1-\rho) \sin \frac{\theta}{2}}{4\left[(1-\rho)^{2}+\sin ^{2} \frac{\theta}{2}\right]} .
$$

The inequality $\sin \theta / 2 \geqslant 1-\rho$ yields

$$
d(\theta) \geqslant \frac{1-\rho}{8 \sin \frac{\theta}{2}} \geqslant \frac{(1-\rho)}{4 \theta} .
$$

Finally, using $\frac{1}{4} \leqslant \rho<1$, we obtain

$$
d(\theta) \leqslant \frac{4(1-\rho)}{\sin \frac{\theta}{2}} \leqslant \frac{4(1-\rho)}{\frac{2}{\pi}\left(\frac{\theta}{2}\right)}=\frac{4 \pi(1-\rho)}{\theta} .
$$

If in (1) and (2) we set $\theta=\pi k / n$, we obtain

$$
\frac{n(1-\rho)}{4 \pi k} \leqslant d\left(\frac{\pi k}{n}\right) \leqslant \frac{4 n(1-\rho)}{k} .
$$

LEMMA 2. If we set $\theta=\pi k / n$, then the hypotheses of Lemma 1 and, in addition, the inequality $\cos \theta \geqslant \rho$ are satisfied for any $\rho, n$, and $k$ satisfying the inequalities $\frac{1}{4} \leqslant \rho<1, n(1-\rho)<1$, and $1 \leqslant k \leqslant \frac{1}{3} n \sqrt{1-\rho}$. 
Proof. Note that $\frac{1}{4}<\rho<1$ implies that $\frac{1}{3} \sqrt{1-\rho}<\frac{1}{2}$ and thus $\theta=\pi k / n<\pi / 2$ for $k$ satisfying $1 \leqslant k \leqslant \frac{1}{3} n \sqrt{1-\rho}$. Hence we have

$$
\begin{aligned}
\cos \theta & >1-\frac{\theta^{2}}{2}=1-\frac{\pi^{2} k^{2}}{2 n^{2}} \geqslant 1-\frac{\pi^{2}}{2 n^{2}}\left(\frac{1}{9} n^{2}(1-\rho)\right) \\
& =1-\frac{\pi^{2}}{18}(1-\rho)>1-(1-\rho)=\rho .
\end{aligned}
$$

Also,

$$
\sin \frac{\theta}{2} \geqslant \frac{2}{\pi} \frac{\theta}{2}=\frac{1}{\pi} \frac{\pi k}{n} \geqslant \frac{1}{n}>1-\rho .
$$

Now, suppose $0<\rho<1$ and set

$$
w=(z-\rho) /(1-\rho z) \text { and } z=(w+\rho) /(1+\rho w) .
$$

Let $\rho \leqslant R<1$. The line $\{w: \operatorname{Re} w=-R\}$ maps onto the circle in the $z$-plane with the points $-(R-\rho) /(1-\rho R)$ and $1 / \rho$ of the real $z$-axis as ends of a diameter. Consider, in the $z$-plane, the chord common to this image circle and the unit circle and let it be of length $2 d$.

LemMa 3. Let $\rho, R$ and $d$ be defined as above. Then

$$
d=\frac{1-\rho^{2}}{1+\rho^{2}-2 \rho R} \sqrt{1-R^{2}}
$$

Proof. Suppose the chord meets the real axis at the point $\zeta$. Then

$$
1-\zeta^{2}=d^{2}=\left(\zeta+\frac{R-\rho}{1-\rho R}\right)\left(\frac{1}{\rho}-\zeta\right)
$$

and hence we find that

$$
\zeta=\frac{2 \rho-R\left(1+\rho^{2}\right)}{1+\rho^{2}-2 \rho R}
$$

Consequently,

$$
d^{2}=\frac{\left(1+\rho^{2}-2 \rho R\right)^{2}-\left(2 \rho-R\left(1+\rho^{2}\right)\right)^{2}}{\left(1+\rho^{2}-2 \rho R\right)^{2}}=\frac{\left(1-R^{2}\right)\left(1-\rho^{2}\right)^{2}}{\left(1+\rho^{2}-2 \rho R\right)^{2}}
$$

and we obtain

$$
d=\frac{1-\rho^{2}}{1+\rho^{2}-2 \rho R} \sqrt{1-R^{2}}
$$

When we use Lemma 3 , we shall set $R=\cos \theta$ where $\theta=\pi k / n$ (compare with Lemma 1).

LEMMa 4. Let $T(z, \rho, n)=[(\rho-z) /(1-\rho z)]^{n}$ and let $S(\rho, n)=\{z:|z|<1$ and $T(z, \rho, n)$ is real $\}$. If $\frac{1}{4}<\rho<1$ and $n(1-\rho)<1$, then $L(\rho, n)>$ $\left[n(1-\rho) \ln \left(\frac{1}{6} n \sqrt{1-\rho}\right)\right] / 4 \pi$ where $L(\rho, n)$ is the length of $S(\rho, n)$. 
Proof. Note that $S(\rho, n)$ consists of the preimages, under the map $(\rho-z) /(1-\rho z)$, of the rays $\left\{w: w=r e^{i \varphi}, 0 \leqslant r<1\right.$ and $\left.\varphi=\pi j / n, 1 \leqslant j \leqslant 2 n\right\}$. For convenience in applying the previous lemmas we relabel these as $\left\{w: w=-r e^{-i \theta}\right.$, $0 \leqslant r<1$, and $\theta=\pi k / n, 1 \leqslant k \leqslant 2 n\}$. These preimages are, of course, just subarcs of circles through $z=\rho$ and orthogonal to $\{|z|=1\}$. The length of such a subarc is greater than the distance of its point of intersection with the unit circle from the real axis. Hence it suffices to estimate the length $d$ of the chords mentioned in the paragraph preceding the statement of Lemma $3(-R=-\cos \theta, \theta=k \pi / n, k=$ $1,2, \ldots, 2 n)$. Using Lemmas 1,2 and 3 , we obtain for $N=\left[\frac{1}{3} n \sqrt{1-\rho}\right]$,

$$
\begin{aligned}
L(\rho, n) & \geqslant \sum_{k=1}^{N} d\left(\frac{\pi k}{n}\right) \geqslant \sum_{k=1}^{N} \frac{n(1-\rho)}{4 \pi k} \\
& =\frac{n(1-\rho)}{4 \pi} \sum_{k=1}^{N} \frac{1}{k} \geqslant \frac{n(1-\rho)}{4 \pi} \ln \left(\frac{1}{6} n \sqrt{1-\rho}\right) .
\end{aligned}
$$

LEMMA 5. Given any $\varepsilon>0$ and any $\delta>0$ there exists an $\alpha>0$ such that $\mid T(z, \rho, n)$ $-1 \mid<\varepsilon$ for $z \in H_{\delta} \equiv\{|z|<1\} \cap\{|z-1| \geqslant \delta\}$ and any $\rho, n$ satisfying $n(1-\rho)<$ $\alpha$.

Proof. Note that $T(-1, \rho, n)=1$ and

$$
T^{\prime}(z, \rho, n)=n(1-\rho)\left(\frac{\rho-z}{1-\rho z}\right)^{n-1} \frac{-1}{(1-\rho z)^{2}} .
$$

Thus $\left|T^{\prime}(z, \rho, n)\right| \leqslant n(1-\rho) \cdot 1 \cdot K_{\delta}$ for $z \in H_{\delta}$. Here $K_{\delta}$ is a constant which depends on $\delta$ only $\left(z \in H_{\delta}, 0<\rho<1\right)$. Thus $|T(z, \rho, n)-1|<n(1-\rho) K_{\delta}$ for $z \in H_{\delta}$ which yields the desired result.

LEMMA 6. Given any $\eta>0$ there exists an $\alpha>0$ such that a connected subset of $S(\rho, n)$ of length $\geqslant\left[n(1-\rho) \ln \left(\frac{1}{6} n \sqrt{1-\rho}\right)\right] / 4 \pi$ (see Lemma 4) is contained in $\{|z-1|<\eta\}$ for $n$ and $\rho$ such that $n(1-\rho)<\alpha$ and $n$ even.

Proof. We shall prove that $\alpha$ may be chosen so that the chords $d_{k}$ whose lengths are estimated in Lemma 4 are contained in $\{|z-1|<\eta\}$. It follows readily that $S(\rho, n)$ is also contained in $\{|z-1|<\eta\}$. Recall that $d_{k} \leqslant 4 n(1-\rho) / k$. Also observe that $d=1$ requires $\zeta=0$ and this in turn requires that $R=2 \rho /\left(1+\rho^{2}\right)$. Thus, $d_{k}$ can be made as small as we like by choosing $n(1-\rho)$ sufficiently small and it remains to show for $k$ satisfying $1 \leqslant k \leqslant \frac{1}{3} n \sqrt{1-\rho}$ that $R_{k}=\cos (\pi k / n) \leqslant$ $2 \rho /\left(1+\rho^{2}\right)$ (so that the chord of length $2 d_{k}$ is "near" $z=1$ rather than $z=-1$ ). It suffices to consider the case $k=1$. To keep the calculations as simple as possible, we shall compare instead $1-\cos ^{2}(\pi k / n)=\sin ^{2}(\pi k / n)$ with $1-\left[2 \rho /\left(1+\rho^{2}\right)\right]^{2}=$ $\left[\left(1-\rho^{2}\right) /\left(1+\rho^{2}\right)\right]^{2}$ and we need to prove that

$$
\sin ^{2}(\pi k / n) \geqslant\left[\left(1-\rho^{2}\right) /\left(1+\rho^{2}\right)\right]^{2} .
$$


Now if $\alpha<\frac{1}{2}$ and $k=1$, we have

$$
\begin{aligned}
\sin ^{2} \theta & \geqslant \frac{4}{\pi^{2}} \cdot \frac{\pi^{2}}{n^{2}} \geqslant 4\left(4(1-\rho)^{2}\right) \\
& \geqslant 16(1-\rho)^{2} \cdot \frac{1}{4}\left(\frac{1+\rho}{1+\rho^{2}}\right)^{2} \geqslant \frac{\left(1-\rho^{2}\right)^{2}}{\left(1+\rho^{2}\right)^{2}}
\end{aligned}
$$

since $\left[(1+\rho) /\left(1+\rho^{2}\right)\right]^{2} \leqslant 4$ if $0<\rho<1$.

Note that the only requirements on $n$ and $\rho$ so that Lemmas 1 through 6 are satisfied are that $\frac{1}{4} \leqslant \rho<1$ and $n(1-\rho)$ is sufficiently small. Thus using Lemmas 1 to 6 and always choosing $n$ to be even, it follows in a straightforward fashion, since $T(1, \rho, n)=1$ for even $n$, that we can construct a convergent Blaschke product $B(z) \equiv \prod_{\nu=1}^{\infty}\left[\left(z-\rho_{\nu}\right) /\left(1-\rho_{\nu} z\right)\right]^{n_{\nu}}$ such that at the $j$ th step the length of the preimage of the segment $(-1,1)$ by the partial product

$$
B_{j}(z) \equiv \prod_{\nu=1}^{j}\left[\left(z-\rho_{\nu}\right) /\left(1-\rho_{\nu} z\right)\right]^{n_{\nu}}
$$

is increased by (see Lemma 4)

$$
\frac{n_{j}\left(1-\rho_{j}\right)}{8 \pi} \ln \left(\frac{1}{6} n_{j} \sqrt{1-\rho_{j}}\right) .
$$

Since (4) equals

$$
\frac{1}{8 \pi}\left[\frac{1}{2} n_{j}\left(1-\rho_{j}\right) \ln \left[n_{j}\left(1-\rho_{j}\right)\right]+\frac{1}{2} n_{j}\left(1-\rho_{j}\right) \ln n_{j}\right],
$$

we can make (5) as large as we like by choosing $n_{j}$ as large as necessary and then choosing $\rho_{j}$ close enough to one to make $n_{j}\left(1-\rho_{j}\right)$ approximately equal to the desired value $\varepsilon_{j}$. It follows easily that $e^{i B(z)}$ has the desired properties.

\section{REFERENCES}

1. C. Belna and G. Piranian, A Blaschke product with a level set of infinite length, Memorial Volume to P. Turan (to appear).

2. J. B. Garnett, F. W. Gehring and P. W. Jones, Level sets for univalent functions and a theorem of Fejer and Riesz (to appear).

3. W. K. Hayman and J.-M. G. Wu, Level sets of univalent functions, Comment. Math. Helv. 56 (1981), 366-403.

4. P. W. Jones, Bounded holomorphic functions with all level sets of infinite length, Michigan Math. J. 27 (1980), 75-80.

5. G. Piranian, Inner functions with a level-set of infinite length, Complex Analysis, Joensuu, 1978 (Proc. Colloq., Univ. Joensuu, Joensuu, 1978), Lecture Notes in Math., vol. 747, Springer-Verlag, Berlin and New York, 1979, pp. 309-313.

6. G. Piranian and A. Weitsman, Level sets of infinite length, Comment. Math. Helv. 53 (1978), 161-164.

Department of Mathematics, Syracuse University, Syracuse, New York 13210 (Current address of K. F. Barth)

Department of MATHEMatics, ImPERIAL College, London SW7 2AZ, UNited Kingdom

Current address (J. G. Clunie): The Open University, Walton Hall, Milton Keynes MH7 6AA, United Kingdom 